\title{
Preconception counselling resource for women with diabetes
}

Aisling Gough, David McCance, Fiona Alderdice, Roy Harper, Valerie Holmes

Queen's University Belfast, Northern Ireland, United Kingdom

\begin{abstract}
Women with diabetes need to plan for pregnancy if they are to reduce their risk of poor pregnancy outcome. While care providers have focused on setting up specialist pre-pregnancy planning clinics to help women prepare for pregnancy, the majority of women do not attend, entering pregnancy unprepared. A major barrier to accessing this care, and a consequence of poor preconception counselling, is a lack of knowledge as to the need to plan and the reasons why. This project addressed an urgent need to raise awareness of the importance of planning for pregnancy among women with diabetes and among the healthcare professionals (HCPs) caring for them. Focus groups with the target groups informed the development of a preconception counselling resource for women with diabetes. Originally produced as a DVD (Diabetes UK funding), this resource has been embedded in routine care in Northern Ireland (NI) since 2010. A subsequent service evaluation of pregnancy planning indicators undertaken across all five antenatal-metabolic clinics in NI indicated that women who viewed the resource were better prepared for pregnancy. In order to increase the positive impact of the resource and to ensure longer term sustainability the DVD was converted to a website, www.womenwithdiabetes.net (Public Health Agency NI funding). The evaluation also highlighted that women with type 2 diabetes were a hard to reach group. As these women are often cared for outside of specialist clinics, it is pertinent that all HCPs caring for women with diabetes are aware of the importance of preconception counselling. Funding also supported the development of an e-learning continuing professional development (CPD) resource within the website. The e-learning resource has since been embedded into existing CPD programmes and is an important tool to ensure that all HCPs caring for women with diabetes are empowered to provide preconception counselling at every opportunity.
\end{abstract}

\section{Problem}

Women with diabetes need to plan for pregnancy if they are to reduce their risk of poor pregnancy outcome. While care providers have focused on setting up specialist pre-pregnancy planning clinics to help women prepare for pregnancy, the majority of women do not attend, entering pregnancy unprepared. Furthermore, women with type 2 diabetes remain a hard to reach group suggesting that a significant problem within primary care in relation to preconception counselling. There is an urgent need to raise awareness of the importance of planning for pregnancy among women and the healthcare professionals caring for them. While this project is based in Northern Ireland, this is a global problem for women with diabetes.

\section{Background}

Women with diabetes have a significantly increased risk of adverse pregnancy outcome, such as congenital malformations and miscarriage, compared with the general maternity population. The risk of adverse outcomes is related to glycaemic control before and during early pregnancy and it follows that pregnancy planning and pre-pregnancy care are associated with reduced risk of adverse pregnancy outcome. Despite the positive impact of pre-pregnancy care the Confidential Enquiry into Maternal and Child Health highlighted that two thirds of women with diabetes received suboptimal preconception care [1]. Focus groups carried out with women with diabetes highlighted that a major barrier to accessing this care, and a consequence of poor preconception counselling, is a lack of knowledge as to the need to plan and the reasons why [2].
This lack of awareness of the risks associated with pregnancy and the role of preconception care in preventing pregnancy complications impacts on the engagement of women with preconception care.

\section{Baseline measurement}

At the start of this project it was clear that women with diabetes were not well prepared for pregnancy. The 2007 Confidential Enquiry into Maternal and Child Health, "Diabetes in pregnancy: are we providing the best care? Findings of a National Enquiry, England, Wales, and Northern Ireland" highlighted that women with diabetes were poorly prepared for pregnancy, with two thirds of women receiving suboptimal preconception care [1]. We carried out focus groups with women with diabetes to decipher the reasons for suboptimal care. We found that while the majority of women were aware of the need to plan pregnancy, few were aware of the rationale for planning [2]. Women were also uncertain about what pre-pregnancy advice entailed. Thus it was evident that while there was a move towards ensuring women had access to pre-pregnancy care clinics there was an urgent need for women to receive guidance about planning pregnancy, and thus the need to attend such clinics, in a motivating, positive, and supportive manner. At the start of this project preconception counselling resources were limited to localised leaflets with no multimedia educational resources available to women in the UK.

\section{Design}

Design and development of the initial DVD centred on two key 
stakeholders, the target group (women with diabetes) and a professional advisory group, working alongside a professional multimedia company [3]. Content for the resource adheres to NICE guidance NG3 Diabetes in Pregnancy [4] and involves women with diabetes sharing their own experiences of planning for pregnancy and their knowledge of issues such as contraception and the reasons why it is so important to plan. As well as featuring in the resource as the 'cast', women with diabetes also provided feedback on style and format. The advisory group ensured content and face validity. Likewise, when the DVD was subsequently converted to an online delivery platform it was important to re-engage with both stakeholders to achieve a highly acceptable resource.

\section{Strategy}

PDSA 1. After initial consultation with the key stakeholders, including the focus groups with women with diabetes, storyboards depicting how the resource would look were presented to both stakeholders. Feedback was given and subsequently acted upon to create a prototype resource ready for evaluation in a pilot study.

PDSA 2. The 52 minute prototype resource was piloted among 16 women with diabetes to ascertain their feedback in relation to comprehensiveness, impact of personal clips, visual attractiveness of screen displays, and perceived relevance. In a separate screening, the 'professional advisory group' ascertained content and face validity (realism). Feedback was considered and changes made as appropriate.

PDSA 3: The resource was evaluated among 97 women with diabetes to determine if it increased knowledge and changed attitudes of women with diabetes towards preconception care. Women were also asked to feedback on the resource; feedback was considered and changes made where appropriate leading to creation of the final DVD, ready for distribution to all women with diabetes in Northern Ireland via their diabetes care teams and GP practices.

PDSA 4: A service evaluation was carried out to establish uptake of the resource post distribution. This revealed that while the majority of women with type 1 diabetes who subsequently became pregnant had received the DVD, only a small proportion of women with type 2 diabetes had received the DVD. This prompted the team to seek alternative means of increasing the impact of the resource and thus the exposure.

PDSA 5. To increase impact of the resource among women the team converted the DVD to a website. Again feedback from both stakeholders (women with diabetes and professional advisory group) was considered within the design process.

PDSA 6. To increase awareness of preconception counselling among healthcare professionals who interact with women with diabetes on a regular basis the team sought to create a version of the website aimed at healthcare professionals. This e-learning website provides HCPs with the tools needed to allow them to talk to women about pregnancy planning.
PDSA 7: As well as raising awareness about the importance of planning for pregnancy the team felt that it was important that women were signposted to pre-pregnancy clinics. Thus within the website details of pre-pregnancy clinics in Northern Ireland were included which would allow women to self refer to begin planning for pregnancy with the support of the multidisciplinary team. It is anticipated that this facility will be expanded to include clinics beyond Northern Ireland.

PDSA 8: With the website developed it was important to raise awareness about the resource among women and HCPs. Within Northern Ireland all GP practices and all community pharmacies were sent packs containing flyers and posters about the resource. Both GPs and pharmacies were encouraged to carry out a trigger for intervention approach with women, whereby either the computer system (GPs) or a script for diabetes related medication (pharmacies) would trigger the HCP to give a flyer about the resource to the woman.

PDSA 9: Finally, the team sought to raise awareness of the resource beyond Northern Ireland and sought to do this by engaging with social media. A Women with Diabetes Twitter account was established @diabeteswomen to reach the target group.

\section{Results}

In a study carried out to explore the impact of the resource on knowledge and attitudes to preconception care (n-97) we demonstrated, using questionnaires pre and post DVD, that the DVD was effective in increasing knowledge and enhancing attitudes of women with diabetes to preconception care. Feedback on the acceptability of the resource was also positive [5]. Furthermore, a recent study in women booking for pregnancy post intervention highlighted improved pregnancy planning indicators in those women who viewed the DVD when compared to those who did not view the DVD [6]. Ultimately, the result of this project is a highly acceptable preconception counselling website for women with diabetes, and for the HCPs caring for them (www.womenwithdiabetes.net). Launched on World Diabetes Day 2014 the website has attracted visitors from 34 countries and has had an average of 4000 hits per month. It is intrinsically linked within other resources, such as continuing professional development packages and the JDRF pregnancy planning tool. The Women with Diabetes twitter account has over 800 followers.

\section{Lessons and limitations}

This project has highlighted the importance of user/patient involvement in the creation of educational resources. Ideas and plans for the resource which the team had discussed prior to the focus groups were altered substantially once focus group feedback was considered. For example, the team had considered the inclusion of video footage of healthcare professionals within the resource advising women on planning pregnancy. Women rejected this idea, instead wanting a real lives approach which led to the resource having a 'cast' of eight women with diabetes. Listening to 
women with diabetes during the design and development phase of both the DVD and the subsequent website development led to the creation of a highly acceptable preconception counselling resource. We faced a major challenge in relation to reaching women with type 2 diabetes, as engagement with primary care was often difficult. To address this we converted the DVD to online delivery, a process which has ensured the longer term sustainability of the resource, ensuring that all women with diabetes can access the resource from anywhere. The addition of the e-learning healthcare professional pages to the website also encourages healthcare professionals to learn about preconception counselling and provides them with the tools necessary to have that all important conversation with women during routine consultations. A targeted poster and flyer campaign was essential to raise awareness about the resource, along with an e-launch utilising the @diabeteswomen twitter account. This multipronged approach at raising awareness was essential to increase website hits.

The project is limited by our inability to test the resource in a randomised controlled trial. Such a study design was not feasible, given that we were seeking to measure outcome of pregnancy. There are inherent difficulties involved in a design that would require randomisation of all non-pregnant women who might subsequently become pregnant, especially given the high rates of unplanned pregnancies. In addition the limited engagement of primary care with research made a regional randomised controlled trial unfeasible.

\section{Conclusion}

Women with diabetes need to plan for pregnancy. This womencentred intervention increases awareness about the need to plan for pregnancy among women and among the HCPs who care for them. By raising awareness of the need to plan and the rationale for planning it is anticipated that attendance at specialist pre-pregnancy clinics will increase, with a subsequent decrease in poor pregnancy outcome.

\section{References}

1. Confidential Enquiry into Maternal and Child Health. Diabetes in Pregnancy: Are we providing the best care? Findings of a National Enquiry: England, Wales and Northern Ireland. London: CEMACH, 2007.

2. Spence M, Alderdice FA, Harper R, McCance DR, Holmes VA. An Exploration of Knowledge and Attitudes Related to Pre-pregnancy Care in Women With Diabetes. Diabetic Medicine 2010; 27:1385-91

3. Spence M, Harper R, McCance DR, Alderdice F, McKinley $M$, Hughes C, Holmes VA. "Women with Diabetes: Things You Need to Know (But Maybe Don't!)"; the systematic development of an innovative DVD to raise awareness of preconception care. European Diabetes Nursing, 2013; 12 : 7-12.

4. National Institute for Clinical Excellence (NICE) (Great Britain): Diabetes in pregnancy: Management of diabetes and its complications from preconception to the postnatal period. London, RCOG Press, 2015 (Available from http://www.nice.org.uk/guidance/ng3 , accessed 28 Sept 2015)

5. Holmes VA, Spence M, McCance DR, Patterson CC, Harper R, Alderdice FA. Evaluation of a DVD for Women with Diabetes: Impact on knowledge and attitudes to preconception care. Diabetic Medicine 2012; 29: 950-6.

6. Holmes VA, Hamill LL, Spence M, Alderdice FA, Patterson CC, Harper R, Loughridge S, McKenna S, McCance DR, for the Women with Diabetes Project Team. Pregnancy planning indicators following the implementation of an educational preconception counselling resource (DVD) for women with diabetes into routine care in Northern Ireland: a Service Evaluation. Diabetic Medicine 2014; 31(Suppl. 1):7

\section{Declaration of interests}

The authors have nothing to declare in terms of competing interests.

\section{Acknowledgements}

Lesley Hamill, Michelle Spence, Chris Patterson, Sarah Loughridge, Sonia McKenna, Michelle McKinley, Mark Davies, John Manderson, Una McErlean, Kieran McGlade, Lynne Thomas, Ciara Mulligan, Adele Kennedy, Neil Black, John Lindsay, Mae McConnell, Oonagh McSorley, Lesley Anderson, Chris Patterson, David Robinson, Clare Hughes, Louisa Dunlop, Brid Farrell, Tracey McCourt, Emma Meneely, Marie King, Diane MacDonald, Katherine Kidd, Florence Findlay-White, Meadhbh Hogg, and Meghan Deery.

Funding: Diabetes UK, Public Health Agency Northern Ireland.

\section{Ethical approval}

The protocol for focus groups work and for the DVD evaluation was approved by the Office for Research Ethics Committees in Northern Ireland (09/NIR/03/71). Part of the study was designed as a service evaluation utilising historic audit data as a comparison group and therefore did not require approval from a Research Ethics Committee. Local approvals were obtained. 